\title{
Counterexamples to Borsuk's Conjecture with Large Girth
}

\author{
R. I. Prosanov ${ }^{1,2 *}$ \\ ${ }^{1}$ Moscow Institute of Physics and Technology (State University), Dolgoprudnyi, \\ Moscow Oblast, 141700 Russia \\ ${ }^{2}$ University of Fribourg, Fribourg, 1700 Switzerland \\ Received March 14, 2018; in final form, March 14, 2018; accepted May 17, 2018
}

\begin{abstract}
Borsuk's celebrated conjecture, which has been disproved, can be stated as follows: in $\mathbb{R}^{n}$, there exist no diameter graphs with chromatic number larger than $n+1$. In this paper, we prove the existence of counterexamples to Borsuk's conjecture which, in addition, have large girth. This study is in the spirit of O'Donnell and Kupavskii, who studied the existence of distance graphs with large girth. We consider both cases of strict and nonstrict diameter graphs. We also prove the existence of counterexamples with large girth to a statement of Lovász concerning distance graphs on the sphere.
\end{abstract}

DOI: $10.1134 / \mathrm{S} 0001434619050249$

Keywords: distance graph, Borsuk's problem.

\section{INTRODUCTION}

In 1933, Borsuk stated the following conjecture, which had become known as Borsuk's conjecture (see [1]): Any set in $\mathbb{R}^{n}$ can be cut into $n+1$ parts of smaller diameter. In spite of strong evidences in favor of this conjecture, it has turned out to be false: in 1993, Kahn and Kalai published the paper [2], in which they constructed finite sets of points which are counterexamples to Borsuk's conjecture. More details on the history of Borsuk's problem can be found in [3]-[8].

By a strict diameter graph we mean a graph whose vertex set is any $S \subset \mathbb{R}^{n}$ and two vertices are joined by an edge if and only if these vertices realize the diameter of the set $S$. A nonstrict diameter graph is any subgraph of a strict diameter graph. Clearly, Borsuk's problem is closely related to the chromatic numbers of such graphs. The existence of finite diameter graphs (not necessarily strict) with chromatic number larger than $n+1$ means that Borsuk's conjecture does not hold.

We also need a slightly more general notion. Suppose given a number $x$. A strict distance graph is a graph whose vertex set is a point set $S \subset \mathbb{R}^{n}$ and two vertices are joined by an edge if and only if the distance between these vertices equals $x$. Similarly, a nonstrict distance graph is any subgraph of a strict distance graph.

The study of distance graphs was strongly motivated by the well-known problem about the chromatic number of Euclidean space. This is the least number of colors sufficient for coloring all points of $\mathbb{R}^{n}$ so that no two points at distance 1 have the same color (see [9]-[16]). As is known, the chromatic number of $\mathbb{R}^{n}$ is attained for a certain finite distance graph [17]. The exact value of the chromatic number is unknown even for the plane. The best estimates presently known are

$$
5 \leq \chi\left(\mathbb{R}^{2}\right) \leq 7
$$

(the lower bound can be found in [18]); for $n$-space, there exists an asymptotic estimate, namely,

$$
(1.239+o(1))^{n} \leq \chi\left(\mathbb{R}^{n}\right) \leq(3+o(1))^{n} .
$$

The lower bound was obtained by Raigorodskii in [19] and the upper one, by Larman and Rogers in [20]. An alternative proof of the upper bound was proposed by the author in [21].

\footnotetext{
*E-mail: rprosanov@mail.ru
} 
In addition to the chromatic number, there is another important characteristic of graphs, girth, which is the length of the shortest cycle. The study of the interplay between the chromatic number and the girth of graphs has a long history. In [22], Erdős proved the existence of graphs with arbitrarily large chromatic number and arbitrarily large girth. In [23], Lovász proposed an explicit construction of such graphs. In [25] and [24], O'Donnell constructed distance graphs in the plane with arbitrarily large girth and chromatic number 4 and, thereby, proved Erdős' conjecture. In [26], Kupavskii studied the asymptotic behavior as $n \rightarrow \infty$ of the chromatic number of distance graphs with girth not smaller than a fixed number $k$ in $\mathbb{R}^{n}$ (see also [27], [28]). In [29], Kupavskii and Polyanskii proved another interesting assertion related to those already mentioned: in $\mathbb{R}^{n}$, a diameter graph with $m$ vertices cannot have more than $m$ cliques of size $n$.

In this paper, we unite these topics by proving the existence of counterexamples with large girth to Borsuk's conjecture. To be more precise, we prove the following theorem.

Theorem 1. For any sufficiently large $n$, there exists a nonstrict diameter graph $G_{n} \subset \mathbb{R}^{n}$ such that $\chi\left(G_{n}\right)>n+1$ and the girth $k\left(G_{n}\right)$ equals $\Omega\left(n^{1 / 4} / \ln ^{3 / 2} n\right)$.

We also give an alternative construction, which makes it possible to construct diameter graphs whose chromatic number grows faster than $n+1$ and odd girth is large (i.e., in these graphs, there are no odd cycles of size smaller than a given value). Nevertheless, the estimate is significantly worse than that of Theorem 1. The advantage of this construction is that the obtained graph is strict.

Theorem 2. For any sufficiently large n, there exists a strict diameter graph $G_{n} \subset \mathbb{R}^{n}$ such that $\chi\left(G_{n}\right)>n+1$ and the odd girth $k_{\text {odd }}\left(G_{n}\right)$ equals $\Omega(\sqrt{\ln n / \ln \ln n})$.

Let us consider another well-known topic related to distance graphs. The chromatic number $\chi\left(S_{r}^{n-1}\right)$ of a sphere of radius $r$ is the least number of colors sufficient for coloring the points of this sphere so that no two points at distance 1 have the same color. Erdös conjectured that, for any fixed radius larger than $1 / 2, \chi\left(S_{r}^{n-1}\right)$ increases with $n$. Lovász proved this in [30] by topological methods; to be more precise, he proved that $\chi\left(S_{r}^{n-1}\right) \geq n$. He also announced in [30] that if $1 / 2<r<\sqrt{n /(2 n+2)}$, then $\chi\left(S_{r}^{n-1}\right) \leq n+1$. This conjecture was disproved in [31] by Raigorodskii, who showed by methods of linear algebra that $\chi\left(S_{r}^{n-1}\right)$ grows exponentially. To this end, he constructed finite distance graphs with large chromatic number in which edges join vertices at distance 1 (we refer to such a distance graph as a unit graph) on the sphere $S_{r}^{n-1}$. The lower bounds were further improved in [32]. We construct counterexamples with large girth to Lovász statement. Namely, we prove the following theorem.

Theorem 3. There exists a sequence of numbers $r_{n}<\sqrt{n /(2 n+2)}$ and a sequence of nonstrict unit graphs $G_{n} \subset S_{r_{n}}^{n-1}$ such that, for each $n, \chi\left(G_{n}\right)>n+1$ and the girth $k\left(G_{n}\right)$ equals $\Omega\left(\sqrt{n} / \ln ^{3 / 2} n\right)$.

However, $r_{n}$ tends to $1 / \sqrt{2}$ with increasing $n$. In this situation, Theorem 2 has the following analog.

Theorem 4. There exists a constant $c^{\prime}$ such that, for $r_{n}=1 / 2+c^{\prime} \sqrt{(\ln n) / n}$, there exists a strict unit graph $G_{n} \subset S_{r_{n}}^{n-1}$ for which $\chi\left(G_{n}\right)>n+1$ and the odd girth $k_{\text {odd }}\left(G_{n}\right)$ equals $\Omega(\sqrt[4]{n / \ln n})$.

In the next section, we give the necessary definitions and all results used in what follows. Then, in Sec. 3, we prove Theorems 1 and 3, after which, in Sec. 4, we prove Theorems 2 and 4. 


\section{PRELIMINARIES TO THE PROOFS}

The construction in the proof of Theorems 1 and 3 is based on the distance graph $G=G(n, v, e)$ whose vertices and edges are defined as follows:

$$
\begin{aligned}
V_{n} & =\left\{\bar{x}=\left(x_{1}, \ldots, x_{n}\right): x_{i} \in\{0,1\}, x_{1}+\cdots+x_{n}=v\right\}, \\
E_{n} & =\{\{\bar{x}, \bar{y}\}:(\bar{x}, \bar{y})=e\},
\end{aligned}
$$

where $(\bar{x}, \bar{y})$ denotes the inner product of the vectors $\bar{x}$ and $\bar{y}$.

In our paper, the key role is played by the following result.

Theorem 5 (Sagdeev [33]). There exists a constant $c$ and functions $v(n)$ and e $(n)$ satisfying the conditions specified below such that, for sufficiently small $\varepsilon$ and $\delta=c \varepsilon^{2} / \ln ^{2} \varepsilon$, any set of at least $(2-\delta)^{n}$ vertices in $G(n, v(n), e(n))$ contains at least $(4-\varepsilon+o(1))^{n}$ edges.

Remark 1. The sequences $v(n)$ and $e(n)$ must satisfy the following conditions:

(1) $\lim _{n \rightarrow \infty}(v(n) / n)=1 / 2$;

(2) $\lim _{n \rightarrow \infty}(e(n) / n)=1 / 4$;

(3) $v(n)-e(n)$ is prime;

(4) $2 e<v$.

The reader can find earlier versions of this theorem and its various geometric corollaries in [34]-[40].

For the proof of Theorem 1, it is important that $v(n)$ and $e(n)$ can be chosen so that

$$
v(n)-\frac{n}{2} \geq e(n)-\frac{n}{4} \text {. }
$$

Note that this is indeed easy to achieve. It suffices, e.g., to take $v(n)=\lfloor n / 2\rfloor$ and define $e(n)$ to be the greatest number not exceeding $\lfloor n / 4\rfloor-1$ and satisfying condition (3) (condition (4) holds automatically). The following assertion is known [41]: There exists a constant $C>0$ such that, for any positive integer $n$, there is a prime between $n$ and $n+C n^{0.525}$. Clearly, for this sequence $e(n)$, condition (2) holds as well.

For the proof of Theorem 3, it is important to achieve that

$$
4 e(n)+n+2<4 v(n) .
$$

This inequality holds for the same $v(n)$ and $e(n)$.

We set $v=v(n)$ and $e=e(n)$. Consider the graph $G=G(n, v, e)$ described above. Note that the number $N$ of its vertices is estimated as

$$
N=\sqrt{\frac{2}{\pi}} \frac{2^{n}}{\sqrt{n}}(1+o(1))
$$

We also use the following results.

Theorem 6 (Kupavskii, [26]). If $G$ is the same distance graph as in Theorem 5 and the inequality $2 /(4-\varepsilon)<2^{-(k-2) /(k-1)}$ holds for some positive integer $k$, then $G$ has a subgraph $G^{\prime}$ with girth at least $k$.

This assertion, although not stated in the paper [26], easily follows from the proof of the main result of that paper.

Theorem 7 (Kupavskii and Raigorodskii, [42]). For a certain constant $c^{\prime}$ and for

$$
r_{n} \geq 1 / 2+c^{\prime} \frac{\ln \ln n}{\ln n)}
$$

(where $n$ is sufficiently large), the sphere $S_{r_{n}}^{n-1} \subset \mathbb{R}^{n}$ of radius $r_{n}$ contains a finite set of diameter 1 which cannot be divided into $n+1$ parts of smaller diameter.

Theorem 8 (Raigorodskii, [31]). For a certain constant $c^{\prime \prime}$ and any $r_{n} \geq 1 / 2+c^{\prime \prime} \sqrt{\ln n / n}$, there exists a strict unit graph $G_{n} \subset S_{r_{n}}^{n-1}$ such that $\chi\left(G_{n}\right)>n+1$.

Now we can prove our theorems. 


\section{PROOF OF THEOREMS 1 AND 3}

Proof of Theorem 1. We fix the following parameters. We set, first, $k=\sqrt{n} /\left(c_{1} \ln ^{3 / 2} n\right)$ for some constant $c_{1}$; second, $\varepsilon=c_{2} / k=c_{1} c_{2} \ln ^{3 / 2} n / \sqrt{n}$; and third,

$$
\delta=2-\frac{2}{\left(c_{3} n\right)^{5 /(2 n)}}, \quad l=(2-\delta)^{n}=\frac{2^{n}}{\left(c_{3} n\right)^{5 / 2}} .
$$

We shall show that, for these $\varepsilon$ and $\delta$, the assumptions of Theorem 5 hold and then we derive the existence of a subgraph $G^{\prime}$ in our graph $G$ which has girth at least $k$ and independence number $\alpha\left(G^{\prime}\right) \leq l$. The latter condition implies the following estimate of the chromatic number of $G^{\prime}$ :

$$
\chi\left(G^{\prime}\right) \geq \frac{N}{l}=\sqrt{\frac{2}{\pi}} \frac{\left(c_{3} n\right)^{5 / 2} 2^{n}(1+o(1))}{2^{n} \sqrt{n}}=n^{2}(1+o(1))
$$

(the constant $c_{3}$ can be set to $(\pi / 2)^{1 / 5}$ ).

Note that

$$
\begin{aligned}
\delta & =\frac{2\left(\left(c_{3} n\right)^{5 /(2 n)}-1\right)}{\left(c_{3} n\right)^{5 /(2 n)}}=\frac{2\left(\exp \left(5 \ln \left(c_{3} n\right) /(2 n)\right)-1\right)}{1+o(1)} \\
& =\frac{2\left(1+5 \ln \left(c_{3} n\right) /(2 n)+O\left(\left(5 \ln \left(c_{3} n\right) /(2 n)\right)^{2}\right)-1\right)}{1+o(1)} \leq \frac{6 \ln n}{n}
\end{aligned}
$$

for sufficiently large $n$.

On the other hand, first, for a certain constant $c_{2}$ and sufficiently large $n$, we have

$$
\frac{2}{4-\varepsilon}<2^{-(k-2) /(k-1)} \text {. }
$$

Indeed,

$$
\begin{aligned}
\frac{2}{4-\varepsilon} & =\frac{1}{2}\left(1+\frac{\varepsilon}{4}+O\left(\varepsilon^{2}\right)\right) \leq \frac{1}{2}+\frac{\varepsilon}{2} \\
2^{-(k-2) /(k-1)} & =\exp \left(-\frac{k-2}{k-1} \ln 2\right)=\exp \left(-\ln 2+\frac{\ln 2}{k-1}\right) \geq \frac{1}{2}+\frac{\ln 2}{2(k-1)} \geq \frac{1}{2}+\frac{\ln 2}{2 k} .
\end{aligned}
$$

Thus, clearly, the required inequality holds for $c_{2}=\ln 2$.

Second, for a certain constant $c_{1}$, we have

$$
c \frac{\varepsilon^{2}}{\ln ^{2} \varepsilon}=\frac{c\left(c_{1} c_{2}\right)^{2} \ln ^{3} n}{n\left(\ln c_{1} c_{2}+(3 / 2) \ln \ln n-(\ln n) / 2\right)^{2}} \geq \frac{6 \ln n}{n} \geq \delta ;
$$

therefore, for these $\varepsilon$ and $\delta$, the assumption of Theorem 5 does indeed hold. Hence Theorem 6 implies the existence of a graph $G^{\prime}$ satisfying the required conditions.

Consider the quadratic equation $n \lambda^{2}-2 v \lambda+e=0$. Note that it has roots. Indeed, according to the remark to Theorem 5, we can choose $v(n)$ and $e(n)$ so that if $\alpha_{n} n=v(n)-n / 2$ and $\beta_{n} n=e(n)-n / 4$, then $\alpha_{n}-\beta_{n} \geq 0$ (in what follows, we omit the subscript $n$ ). The discriminant of this equation (divided by 4 ) equals

$$
v^{2}-e n=\left(\frac{n}{2}+\alpha n\right)^{2}-\left(\frac{n}{4}+\beta n\right) n=\left(\alpha-\beta+\alpha^{2}\right) n^{2} \geq 0 .
$$

Take a root $\lambda$ of this equation. Let $\bar{x}$ be a vertex of the graph $G$. We set $\widetilde{x}_{i}=x_{i}-\lambda$ and consider the vector

$$
\widetilde{\bar{x}} * \widetilde{\bar{x}}=\left(\widetilde{x}_{1}^{2}, \widetilde{x}_{1} \widetilde{x}_{2}, \ldots, \widetilde{x}_{n}^{2}\right) .
$$

This vector lies in a subspace of dimension $n(n+1) / 2$ in $\mathbb{R}^{n^{2}}$. Obviously, if $\lambda \neq 1 / 2$, then the map $\bar{x} \rightarrow \widetilde{\bar{x}} * \widetilde{\bar{x}}$ is injective. Clearly, $1 / 2$ is a unique root of the trinomial specified above only if $v=n / 2$ and $e=n / 4$, but it is clear from the remark to Theorem 5 that this cannot happen. Let $G^{\prime \prime}$ be the graph 
whose vertices are the images of those of $G^{\prime}$ and the edges are defined accordingly. Let us show that this is a diameter graph.

The vectors in the given set satisfy the relation $(\widetilde{\bar{x}} * \widetilde{\bar{x}}, \widetilde{\bar{y}} * \widetilde{\bar{y}})=\left((\bar{x}, \bar{y})-2 v \lambda+n \lambda^{2}\right)^{2}$. If $(\bar{x}, \bar{y})=e$, then this inner product vanishes (and attains its minimum, because this is a perfect square). On the other hand, we have

$$
|\widetilde{\bar{x}} * \widetilde{\bar{x}}-\widetilde{\bar{y}} * \widetilde{\bar{y}}|^{2}=(\widetilde{\bar{x}} * \widetilde{\bar{x}}, \widetilde{\bar{x}} * \widetilde{\bar{x}})+(\widetilde{\bar{y}} * \widetilde{\bar{y}}, \widetilde{\bar{y}} * \widetilde{\bar{y}})-2(\widetilde{\bar{x}} * \widetilde{\bar{x}}, \widetilde{\bar{y}} * \widetilde{\bar{y}}) .
$$

Since the lengths $\widetilde{\bar{x}} * \widetilde{\bar{x}}$ and $\widetilde{\bar{y}} * \widetilde{\bar{y}}$ are fixed, it follows that this expression attains its maximum when the inner product is minimum. Therefore, the edges of the graph $G^{\prime}$ correspond to those pairs of vertices of $G^{\prime \prime}$ at which the diameter of $G^{\prime \prime}$ is realized.

We obtain the diameter graph $G^{\prime \prime}$ in dimension $m=n(n+1) / 2 \sim n^{2} / 2$ whose chromatic number equals $n^{2}(1+o(1))=2 m(1+o(1))>m+1$ for sufficiently large $m$ and whose girth equals

$$
\Omega\left(\frac{\sqrt{n}}{\ln ^{3 / 2} n}\right)=\Omega\left(\frac{m^{1 / 4}}{\ln ^{3 / 2} m}\right) .
$$

Note that Theorem 6 gives a nonstrict distance graph: not all pairs of vertices at a given distance are joined by edges. Accordingly, the diameter graph obtained by us is nonstrict as well.

Given any other $m$, we choose the greatest $n$ for which $n(n+1) / 2 \leq m$ and consider $G^{\prime \prime}$ embedded in any $n(n+1) / 2$-plane in $\mathbb{R}^{m}$. Since $m<(n+1)(n+2) / 2$ and

$$
\chi\left(G^{\prime \prime}\right)=n^{2}(1+o(1))>\frac{(n+1)(n+2)}{2} \geq m+1
$$

for sufficiently large $n$, it follows that this graph is a counterexample to Borsuk's conjecture. Clearly, for this $m$, its girth is also estimated as $\Omega\left(m^{1 / 4} / \ln ^{3 / 2} m\right)$. This completes the proof of the theorem.

Proof of Theorem 3. The proof of Theorem 3 is similar to that of Theorem 1 with only a minor difference in the choice of parameters. We set

$$
k=\frac{\sqrt{n}}{c_{1} \ln ^{3 / 2} n}, \quad \varepsilon=\frac{c_{2}}{k}=\frac{c_{1} c_{2} \ln ^{3 / 2} n}{\sqrt{n}}, \quad \delta=2-\frac{2}{\left(c_{4} n\right)^{3 /(2 n)}}, \quad l=(2-\delta)^{n}=\frac{2^{n}}{\left(c_{4} n\right)^{3 / 2}} .
$$

It is easy to see that all assumptions of Theorems 5 and 6 again hold, so that, under an appropriate choice of the constant $c_{4}$, there exists a graph $G^{\prime}$ with girth at least $k$ and chromatic number $\chi\left(G^{\prime}\right) \geq 2 n(1+o(1))$, which is greater than $n+1$ for sufficiently large $n$.

Let us show that this graph is isomorphic to a unit graph on a sphere of small radius. Note that all vertices of $G^{\prime}$ lie on the sphere of radius $\sqrt{n} / 2$ centered at $(1 / 2,1 / 2, \ldots, 1 / 2)$, and the squared distance between any two vertices in $G^{\prime}$ equals $2 v-2 e$. Performing a homothety of this sphere such that the distance between any two vertices forming an edge becomes equal to 1 , we obtain a sphere of radius $\sqrt{n} /(2 \sqrt{2(v-e)})$. According to the remark to Theorem 5 , we can assume that $4 e+n+2<4 v$. Then, as can easily be seen, the radius of the obtained sphere is less than $\sqrt{n /(2 n+2)}$.

\section{PROOF OF THEOREMS 2 AND 4}

Proof of Theorem 2. Consider a diameter graph $G$ whose vertices are the points of the finite set from Theorem 7 and edges join pairs of vertices at which the diameter is realized; then $\chi(G)>n+1$. Let us show that the odd girth of this graph is not smaller than $(\pi / 4) \sqrt{\ln n /\left(c^{\prime} \ln \ln n\right)}$.

Let $\bar{u} \in S_{r}^{n-1}$. Consider the set of points lying on $S_{r}^{n-1}$ at distance 1 from $\bar{u}$. This is the boundary of a spherical cap of spherical radius $\phi$ centered at $\bar{v}=-\bar{u}$; we denote it by $\widetilde{S}(\bar{v}, \phi)$. Note that all points at distance 1 from at least one point in $\widetilde{S}(\bar{v}, \phi)$ belong to the hat $\widetilde{S}(\bar{u}, 2 \phi)$, all points at distance 1 from at least one of these points belong to $\widetilde{S}(\bar{v}, 3 \phi)$, and so on. Thus, clearly, a cycle of length $2 l+1$ can be realized as a graph of unit diameter on the sphere $S_{r}^{n-1}$ only if the caps $\widetilde{S}(\bar{u},(l+1) \phi)$ and $\widetilde{S}(\bar{v}, l \phi)$ 
have a common point (and, hence, cover the entire sphere). This can happen only if $(2 l+1) \phi \geq \pi$, i.e., $\phi \geq \pi /(2 l+1)$. Note that

$$
\cos \frac{\phi}{2}=\frac{1}{2 r} \geq 1-\frac{2 c \ln \ln n}{\ln n} .
$$

Moreover,

$$
\cos \frac{\pi}{2 l+1}=1-\frac{\pi^{2}}{(2 l+1)^{2}}+O\left(\frac{1}{(2 l+1)^{4}}\right) \leq 1-\frac{\pi^{2}}{2(2 l+1)^{2}} .
$$

We see that if $2 l+1<(\pi / 4) \sqrt{\ln n /\left(c^{\prime} \ln \ln n\right)}$, then

$$
\phi<\frac{\pi}{2 l+1} ;
$$

therefore, no cycle of length $2 l+1$ fits into this sphere. Hence, indeed, the odd girth of the graph $G$ is not smaller than $(\pi / 4) \sqrt{\ln n /\left(c^{\prime} \ln \ln n\right)}$, as required.

Proof of Theorem 4. Theorem 4 naturally follows from Theorem 8 and the observation that an odd cycle of length smaller than $(\pi / 4) \sqrt[4]{n /\left(c^{\prime \prime 2} \ln n\right)}$ cannot fit into a sphere of such radius; this is shown in the same way as in the proof of Theorem 3 .

\section{FUNDING}

This work was supported by the program "Leading Scientific Schools" under grant NSh-6760.2018.1 and by the Swiss National Science Foundation under grant SNSF-200021_169391.

\section{REFERENCES}

1. K. Borsuk, "Drei Sätze über die n-dimensionale euklidische Sphäre," Fundam. Math. 20, 177-190 (1933).

2. J. Kahn and G. Kalai, "A counterexample to Borsuk's conjecture," Bull. Amer. Math. Soc. (N. S.) 29 (1), 60-62 (1993).

3. P. Brass, W. Moser, and J. Pach, Research Problems in Discrete Geometry (Springer-Verlag, New York, 2005).

4. V. G. Boltyanski, H. Martini, and P. S. Soltan, Excursions into Combinatorial Geometry (Springer-Verlag, Berlin, 1997).

5. A. M. Raigorodskii, "Borsuk's problem and the chromatic numbers of some metric spaces," Uspekhi Mat. Nauk 56 (1 (337)), 107-146 (2001) [Russian Math. Surveys 56 (1), 103-139 (2001)].

6. A. M. Raigorodskii, "Around Borsuk's hypothesis," Sovrem. Mat., Fundam. Napravl. 23, 147-164 (2007) [J. Math. Sci. 154 (4), 604-623 (2008)].

7. A. M. Raigorodskii, "Three lectures on the Borsuk partition problem," in Surveys in Contemporary Mathematics, London Math. Soc. Lecture Note Ser. (Cambridge Univ. Press, Cambridge, 2007), Vol. 347, pp. $202-248$.

8. A. M. Raigorodskii, "Combinatorial geometry and coding theory," Fund. Inform. 145 (3), 359-369 (2016).

9. A. Soifer, The Mathematical Coloring Book: Mathematics of Coloring and the Colorful Life of its Creators (Springer-Verlag, New York, 2008).

10. A. M. Raigorodskii, "Coloring distance graphs and diameter graphs," in Thirty Essays on Geometric Graph Theory (Springer-Verlag, New York, 2013), pp. 429-460.

11. A. M. Raigorodskii, "Cliques and cycles in distance graphs and diameter graphs," in Discrete Geometry and Algebraic Combinatorics, Contemp. Math. (Amer. Math. Soc., Providence, RI, 2014), Vol. 625, pp. 93-109.

12. D. D. Cherkashin and A. M. Raigorodskii, "On the chromatic numbers of low-dimensional spaces," Dokl. Ross. Akad. Nauk 472 (1), 11-12 (2017) [Dokl. Math. 95(1), 5-6(2017)].

13. D. Cherkashin, A. Kulikov, and A. Raigorodskii, "On the chromatic numbers of small-dimensional Euclidean spaces,” Discrete Appl. Math. 243, 125-131 (2018).

14. L. I. Bogolyubskii and A. M. Raigorodskii, "On the measurable chromatic number of a space of dimension $n \leq 24$," Dokl. Ross. Akad. Nauk 465 (6), 647-650 (2015) [Dokl. Math. 92 (3), 761-763 (2015)].

15. L. I. Bogolyubskii and A. M. Raigorodskii, "A remark on lower bounds for the chromatic numbers of spaces of small dimension with metrics $\ell_{1}$ and $\ell_{2}$," Mat. Zametki 105 (2), 187-213 (2019) [Math. Notes 105 (2), 180-203 (2019)]. 
16. A. Ya. Kanel-Belov, V. A. Voronov, and D. D. Cherkashin, "On the chromatic number of an infinitesimal plane layer," Algebra Anal. 29 (5), 68-89 (2017) [St. Petersbg. Math. J. 29 (5), 761-775 (2018)].

17. N. G. de Bruijn and P. Erdős, "A colour problem for infinite graphs and a problem in the theory of relations," Nederl. Akad. Wetensch. Proc. Ser. A 54, 369-373 (1951).

18. A. D. N. J. de Grey, "The chromatic number of the plane is at least 5," Geombinatorics 28 (1), 18-31 (2018).

19. A. M. Raigorodskii, "On the chromatic number of a space," Uspekhi Mat. Nauk 55 (2(332)), 147-148 (2000) [Russian Math. Surveys 55 (2), 351-352 (2000)].

20. D. G. Larman and C. A. Rogers, "The realization of distances within sets in Euclidean space," Mathematika 19, 1-24 (1972).

21. R. Prosanov, A New Proof of the Larman-Rogers Upper Bound for the Chromatic Number of the Euclidean Space, 1610.02846 (2016).

22. P. Erdős, "Graph theory and probability," Canad. J. Math. 11, 34-38 (1959).

23. L. Lovász, "On chromatic number of finite set-systems," Acta Math. Acad. Sci. Hungar. 19, 59-67 (1968).

24. P. O'Donnell, "Arbitrary girth, 4-chromatic unit distance graphs in the plane I. Graph description," Geombinatorics 9 (3), 145-152 (2000).

25. P. O'Donnell, "Arbitrary girth, 4-chromatic unit distance graphs in the plane II. Graph embedding," Geombinatorics 9 (4), 180-193 (2000).

26. A. B. Kupavskii, "Distance graphs with large chromatic number and arbitrary girth,” Mosc. J. Comb. Number Theory 2 (2), 52-62 (2012).

27. A. B. Kupavskii, "Explicit and probabilistic constructions of distance graphs with small clique numbers and large chromatic numbers," Izv. Ross. Akad. Nauk Ser. Mat. 78 (1), 65-98 (2014) [Izv. Math. 78 (1), 59-89 (2014)].

28. Yu. A. Demidovich, "Lower bound for the chromatic number of a rational space with metric $l_{u}$ and with one forbidden distance," Mat. Zametki 102 (4), 532-548 (2017) [Math. Notes 102 (4), 492-507 (2017)].

29. A. B. Kupavskii and A. Polyanskii, "Proof of Schur's conjecture in $\mathbb{R}^{D}$," Combinatorica 37 (6), 1181-1205 (2017).

30. L. Lovász, "Self-dual polytopes and the chromatic number of distance graphs on the sphere," Acta Sci. Math. (Szeged) 45 (1-4), 317-323 (1983).

31. A. M. Raigorodskii, "On the chromatic numbers of spheres in $\mathbb{R}^{n}$," Combinatorica 32 (1), 111-123 (2012).

32. O. A. Kostina and A. M. Raigorodskii, "On lower bounds for the chromatic number of sphere," Dokl. Ross. Akad. Nauk 463 (6), 639-641 (2015) [Dokl. Math. 92 (1), 500-502 (2015)].

33. A. A. Sagdeev, "On the Frankl-Rödl theorem," Izv. Ross. Akad. Nauk Ser. Mat. 82 (6), 128-157 (2018) [Izv. Math. $82(6), 1196-1224(2018)]$.

34. A. M. Raigorodskii and A. A. Sagdeev, "On the chromatic number of a space with a forbidden regular simplex,” Dokl. Ross. Akad. Nauk 472 (2), 127-129 (2017) [Dokl. Math. 95 (1), 15-16 (2017)].

35. A. A. Sagdeev, "Lower bounds for the chromatic numbers of distance graphs with large girth," Mat. Zametki 101 (3), 430-445 (2017) [Math. Notes 101 (3), 515-528 (2017)].

36. R. I. Prosanov, A. M. Raigorodskii, and A. A. Sagdeev, "Improvement of the Frankl-Rödl theorem and geometric consequences,” Dokl. Ross. Akad. Nauk 475 (2), 137-139 (2017) [Dokl. Math. 96 (1), 336-338 (2017)].

37. A. A. Sagdeev, "On a Frankl-Rödl theorem and its geometric corollaries," Electron. Notes in Discrete Math. 61, 1033-1037 (2017).

38. A. A. Sagdeev, "The chromatic number of space with forbidden regular simplex," Mat. Zametki 102 (4), 579-585 (2017) [Math. Notes 102 (4), 541-546 (2017)].

39. A. A. Sagdeev, "Improved Frankl-Rödl theorem and some of its geometric consequences," Problemy Peredachi Informatsii 54 (2), 45-72 (2018) [Problems Inform. Transmission 54 (2), 139-164 (2018)].

40. A. A. Sagdeev, "Exponentially Ramsey sets," Problemy Peredachi Informatsii 54 (4), 82-109 (2018) [Problems Inform. Transmission 54 (4), 372-396 (2018)].

41. R. C. Baker, G. Harman, and J. Pintz, "The difference between consecutive primes. II," Proc. London Math. Soc. (3) 83 (3), 532-562 (2001).

42. A. B Kupavskii and A. M. Raigorodskii, "Counterexamples to Borsuk's conjecture on spheres of small radii," Mosc. J. Comb. Number Theory 2 (4), 27-48 (2012). 